\title{
Hypotheses on the role of the protistan rare biosphere in a changing world
}

\author{
David A. Caron*, Peter D. Countway \\ Department of Biological Sciences, University of Southern California, 3616 Trousdale Parkway, AHF 301, Los Angeles, \\ California 90089-0371, USA
}

\begin{abstract}
Studies of protistan diversity have gained impetus in microbial ecology during the last decade due primarily to the widespread application of molecular approaches developed initially to study bacteria, archaea and viruses. These investigations have revealed many undescribed taxa and lineages of protists, and have indicated the presence of large numbers of rare taxa (the microbial rare biosphere) among the relatively few abundant species that dominate these assemblages in any particular environment. As a consequence of this incredible diversity, the species richness of most natural protistan assemblages is still poorly characterized. One question that has arisen from these studies is whether the taxa comprising the rare biosphere play significant roles in community function, or merely represent inactive, moribund or dead individuals that have little or no ecological importance. We hypothesize that many of the protistan rare taxa can and do become dominant with changing environmental conditions, and that the ecological redundancy represented by these species indicates a highly adaptable microbial eukaryotic community that can maintain biogeochemical processes during significant and perhaps dramatic shifts in environmental forcing factors. If true, the structure of protistan assemblages (and presumably other microbial assemblages) may act to buffer the impact of changing environmental conditions by preventing wholesale changes in basic ecosystem processes. However, it is not clear whether many of the species that might dominate under altered environmental conditions (e.g. toxic or noxious species of protists) would support the same macrobial assemblages that existed previously in those environments.
\end{abstract}

KEY WORDS: Protists · Diversity $\cdot$ Community composition $\cdot$ Biogeography $\cdot$ Ecosystem function

Resale or republication not permitted without written consent of the publisher

\section{INTRODUCTION}

Microbial community structure is highly responsive to environmental forcing. The complexity and ephemeral nature of the taxonomic composition of the bacterial, archaeal, viral and eukaryal assemblages have been and continue to be major impediments in characterizing these communities and predicting their behaviors. Dramatic changes in environmental parameters will cause massive shifts in microbial species composition, but even apparently slight changes in environmental conditions have long been known to bring about sometimes dramatic changes in taxonomic composition. Indeed, classical studies from more than half a century ago noted the significant effects on the bacterial community by merely placing water samples in bottles (Zobell 1943).
Less information is available regarding the response of microbial community processes to changes in environmental conditions, but it is generally assumed that these activities are much more stable than taxonomic composition. Measurements of a number of rate processes (photosynthesis, bacterivory, herbivory, respiration and nutrient remineralization) are still routinely conducted by following changes in water samples held in containers and incubated for periods of time ranging from a few hours to a few days (Ferrier-Pagès \& Rassoulzadegan 1994, Landry et al. 1995, Falkowski \& Raven 1997, Caron 2000, Sherr \& Sherr 2002). It is anticipated that microbial community structure changes substantially in the bottles during these incubations (based on long-standing knowledge noted above), but the underlying assumption is that the rates of microbial activities are similar to the rates of these 
processes in the surrounding water, and thus these experiments provide a reasonable estimate of rates in nature. Bottle incubations can possess artifacts that might lead one to be skeptical of data generated by these techniques (Dolan \& McKeon 2005, Agis et al. 2007). Nevertheless, there is general consensus by most investigators that these experimental approaches yield credible estimates of community activities, and there are presently few alternative methodologies. If rates are generally unaffected by containment, one can logically question what attribute of a microbial community allows it to maintain relatively stable ecosystem function in the face of ever changing taxonomic structure.

Recent studies have begun to shed light on one possible explanation for this apparent conundrum. The application of molecular biological approaches to assess the diversity of natural microbial assemblages has revealed an incredible diversity among all types of microbes (DeLong 1992, Fuhrman et al. 1992, Massana et al. 2002, Stoeck et al. 2003, Romari \& Vaulot 2004, Lovejoy et al. 2006, Countway et al. 2007, Vaulot et al. 2008). Large numbers of undescribed taxa, and even new lineages, have been discovered. Furthermore, rank abundance curves (graphical representations of the abundances of microbial taxa present in an assemblage) generated from these studies have demonstrated the presence of a huge number of taxa present at extremely small percentages of the total abundance of microbes in virtually all communities examined. Information is now emerging on the ubiquity and remarkable number of species comprising this 'rare biosphere' of microbes globally in aquatic and soil ecosystems (Countway et al. 2005, Pedrós-Alió 2006, Sogin et al. 2006, Pedrós-Alió 2007, Elshahed et al. 2008).

The extent of the diversity contained in the microbial rare biosphere is presently unknown, and questions remain regarding its potential ecological importance, if any. Towards this end, we propose that many species comprising the rare biosphere are functionally similar but competitively inferior to the taxa that dominate a given microbial community at a particular time, place and set of environmental and biological conditions. We hypothesize, however, that rare species have tremendous importance because many are responsive to minor changes in environmental conditions that might disfavor the abundant taxa and favor some of the rare taxa, resulting in rapid and frequent reassemblies of the taxonomic composition of the microbial community. Finally, we suggest that these reassemblies often take place without substantive changes in the rates and types of basic processes conducted by microbes. As a consequence, the microbial rare biosphere constitutes a reservoir of taxa that provides biological buffering capacity, allowing relatively stable community function over broad ranges of environmental forcing factors.

\section{THE MICROBIAL RARE BIOSPHERE}

\section{What is the rare biosphere?}

The term 'rare biosphere' refers to the vast numbers of taxa that are present at extremely low abundances in most natural microbial assemblages (including protistan assemblages; Fig. 1). This concept has emerged in the recent literature primarily as a consequence of numerous surveys of microbial diversity that have employed DNA sequence-based approaches (Sogin et al. 2006). The presence of large numbers of rare microbial taxa in most environments has made it exceedingly difficult to assess local species richness using the approaches commonly employed for this purpose (Schloss \& Handelsman 2004, Countway et al. 2005, Epstein \& López-García 2008). Sequencing of many individual clones in clone libraries or, more recently, high-throughput sequencing approaches (Huse et al. 2008) have documented the presence of relatively few common taxa but vast numbers of taxa that comprise only one or a few sequences of a library or sample. An exact definition of the term rare biosphere has been elusive, but a growing number of researchers now use the term to describe a constantly changing collection of rare microbial taxa that is persistently present in the vast majority of ecosystems examined to date. The concept is not restricted to any specific microbial group or to aquatic communities. Soil ecosystems also contain many rare sequence types among a small number of dominant sequences (Elshahed et al. 2008).

Awareness has grown rapidly with respect to the existence and magnitude of the rare biosphere, and a number of questions have emerged regarding the source, composition, ubiquity and persistence of this component of microbial assemblages. Is a significant fraction of the rare biosphere composed of dead cells or molecular debris rather than viable microorganisms? Are viable rare taxa present as active cells or nonactive resting stages (e.g. cysts, spores)? The demonstration of many rare taxa in natural samples has relied largely on taxonomically informative DNA sequences, and it is presently unclear what percentage of these sequences might be attributed to dead cells or free DNA. It is probable that some of these sequences are derived from non-living or non-active cells. Differences have been observed between rDNA and rRNA clone libraries generated from the same sample (Stoeck et al. 2007), but there are presently no quantitative estimates of the contribution of non-living material to environmental clone libraries. 


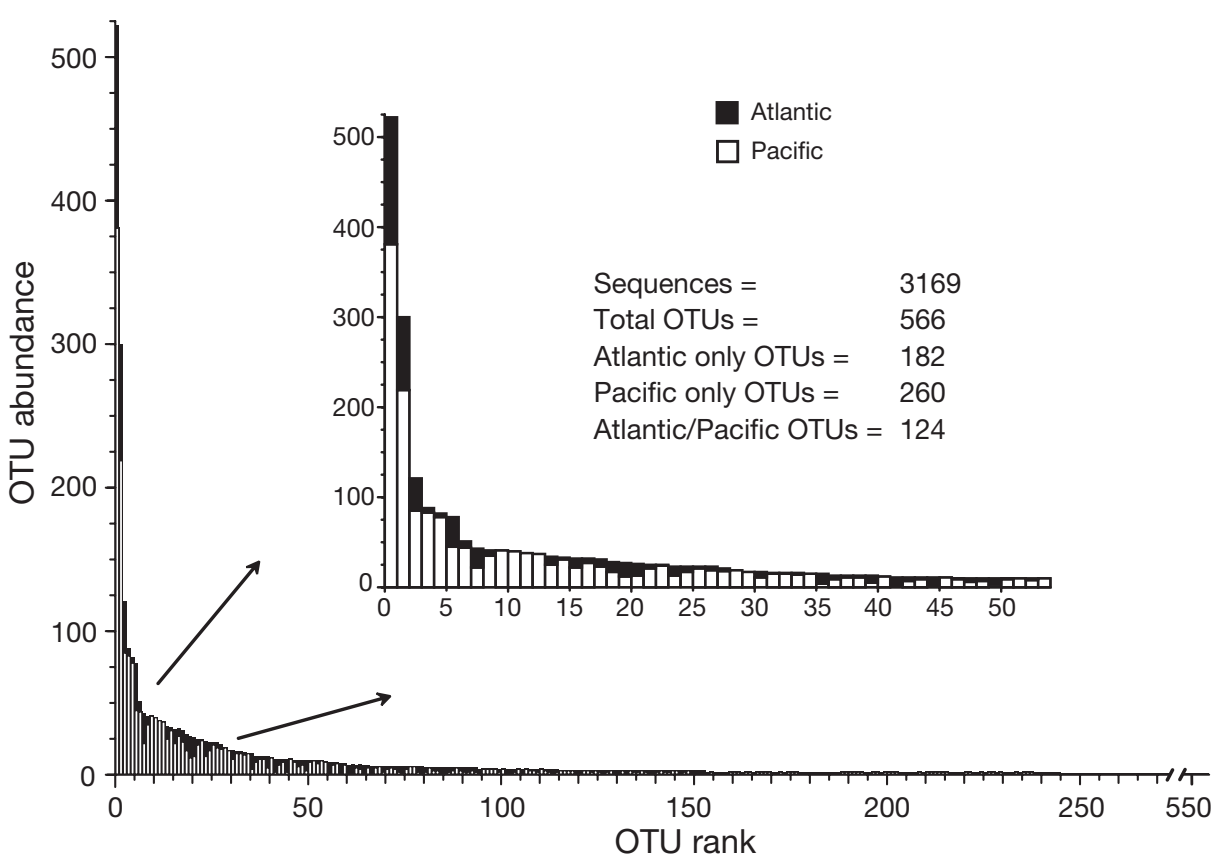

Fig. 1. Rank abundance curve of protistan operational taxonomic units (OTUs) for a combined data set of $>3000$ partial $18 \mathrm{~S}$ rRNA gene sequences from the North Atlantic and North Pacific oceans. Atlantic samples were collected during a single cruise from multiple depths at 2 stations in the western Sargasso Sea and 1 in the Gulf Stream. See Countway et al. (2007) for details. These samples included integrated euphotic zone samples $(15$ and $105 \mathrm{~m})$ and samples collected at $2500 \mathrm{~m}$. Pacific samples were collected from 4 depths (5 m, chlorophyll max, 150 and $500 \mathrm{~m}$ ) across 4 seasons (Jan, Apr, Jul and Oct) at a single coastal site in the San Pedro Channel located approximately halfway between Santa Catalina Island and the coast of California $\left(33^{\circ} 33^{\prime} \mathrm{N}\right.$ $118^{\circ} 24^{\prime} \mathrm{W}$; total water depth approximately $895 \mathrm{~m}$ ). The original analysis consisted of 4051 sequences (1095 Atlantic, 2956 Pacific) distributed among 601 OTUs. Subtraction of non-protistan OTUs (metazoa) reduced the total number to the 566 protistan OTUs that appear in this figure. Many rare protistan OTUs from both oceans contributed to 'Atlantic only' or 'Pacific only' OTUs, with only $22 \%$ of the total OTUs detected in both the Atlantic and Pacific Ocean samples. Unequal sampling effort contributed to the higher abundance of 'Pacific only' OTUs. The most abundant OTUs ( $\geq 10$ members) comprised only $10 \%$ of the 566 OTUs, but collectively they comprised $70 \%$ of the sequences. Many of the most abundant OTUs were found in both Atlantic and Pacific clone libraries. Sampling details and DNA protocols are provided in Countway (2005) and Countway et al. (2007). OTU calling was based on the procedure detailed in Caron et al. (2009)

If rare taxa are alive and active in microbial communities, what is the nature of their existence? Do these species play important ecological roles at very low abundances (such as apex predators), do they represent expatriated individuals from distant endemic sites that are dying out in a new locale, or are they endemic species that are competitively inferior to other endemic taxa under the environmental conditions at that particular time? Alternatively, are rare taxa abundant and active taxa in microenvironments within an ecosystem, and their rare status is merely a consequence of the fact that these microenvironments are rare, and therefore routine sampling approaches collect relatively few of them in any given sample?

The answers to any or all of these questions may be 'yes' for different taxa within the rare biosphere and may have different degrees of applicability for different microbial groups (archaea, bacteria, viruses and protists) due to differences in life cycles, dispersal and resilience to changes in environmental conditions. Regardless of the specific nature(s) of the microbes composing the rare biosphere, the taxonomic diversity contributed by the rare taxa is stunning (Fig. 1).

\section{Ecological roles for the rare biosphere?}

We hypothesize that the enormous diversity of rare microbial taxa indicates the existence of a community structure for microbes that is different than the one classically conceptualized for communities of macroorganisms. Population sizes of aquatic microbial assemblages (including most protistan taxa) are enormous (Fenchel \& Finlay 2003, 2004), and their small cell sizes may facilitate dispersal over great distances. It has been proposed that these features result in very high local diversity but lower global diversity for protists relative to macroorganisms (Finlay \& Clarke 1999b, 
Finlay 2002, Finlay et al. 2004, Green et al. 2004, Green \& Bohannan 2006), although this issue is still being debated (Fenchel \& Finlay 2004, Foissner 2006, Caron 2009).

It is conceivable that large population size and highly effective dispersal of microbes thwart or dampen the outcome of resource competition, predator-prey relationships and other interactions among microbes. No matter how superior, competitive dominants probably never completely eliminate competitively inferior protistan taxa. Rare species capable of even meager growth rates may be able to counter very low mortality losses that might be expected for rare taxa. If extremely low abundance provides a perfect refuge from grazing mortality, taxa may persist simply by withstanding starvation conditions via resting stages or reduced metabolic activity (Fenchel \& Finlay 1983, Caron et al. 1990). Given that environmental conditions in most natural ecosystems change often, rare taxa might also be maintained by experiencing bursts of population growth during short periods of favorable conditions. Alternatively, local extinction might occur in some habitats, but periodic reintroduction from distant locales may effectively maintain populations at very low absolute abundance.

Collectively, these mechanisms for creating and maintaining a rare biosphere imply that it is probable that a tremendous number of microbial taxa will be present at any given locale. In turn, the presence of these rare taxa provides a vast collection of species that might be responsive to changes in environmental conditions and increase rapidly in abundance if chemical, physical or biological conditions change in their favor. This hypothesis conceptualizes the rare biosphere as a continually changing assortment of taxa, rather than a static collection of species that are always rare. As selection for or against particular species changes, they shift from being rare to common or vice versa.

We further hypothesize that substantial changes in the taxonomic composition of microbial assemblages may alter the specific pathways of energy and elemental flow in marine food webs, but that these rearrangements may take place in the relative absence of changes in community-level processes (total system photosynthesis, respiration, elemental cycling, etc.). This hypothesis implies the existence of guilds of microbial species that play similar ecological roles but possess somewhat different optima for environmental conditions or other ecosystem features. The presumption of near-redundancy of ecological roles among microbial species is not a new concept (Dolan 1991, Dolan et al. 2009), but we are not aware of it having been proposed specifically in reference to the possible ecological roles of the rare biosphere of microbial taxa.
Our concept of rapid community reassembly of microbial assemblages is depicted diagrammatically in Fig. 2. A hypothetical microbial assemblage displaying a typical rank abundance curve (Panel 1) and exposed to changes in environmental conditions leads to a shift in the selective forces acting on the microbial taxa, resulting in positive net population growth of some rare taxa and negative net growth of some abundant taxa (Panel 2). The rank abundance curve of the reassembled community retains the same shape (Panel 3), but with different dominant and rare taxa than the original assemblage. Additional changes in environmental conditions result in subsequent community reassemblies (Panel 4) and communities with similarly shaped rank abundance curves but different dominant and rare taxa (Panel 5). Finally, we hypothesize that, throughout these changes in community structure, community function may remain relatively unchanged if rare taxa favored by the environmental changes fulfill similar ecological roles as the abundant species that were displaced (Panel 6).

Guilds of protistan species with similar ecological function might include taxa that have experienced convergent evolution due to limited niche space (e.g. nutrient preferences among photosynthetic taxa or prey preferences among heterotrophs). Alternatively, guilds might represent closely related species that differ in their optimal environmental conditions for growth, or even strain-strain differences within species. Relatively few studies of protistan population genetics have been conducted to date, but the available information indicates substantial levels of genetic and physiological diversity within some populations (Rynearson \& Armbrust 2004, Shankle et al. 2004, Boenigk et al. 2005, Evans et al. 2005, Rynearson et al. 2006, Alpermann et al. 2009). Large numbers of genetic variants might provide a reservoir of taxa with similar overall ecological function but slightly different environmental preferences or susceptibilities to predation or viral infection.

There is still considerable debate among protistan taxonomists whether much of the DNA sequence diversity in natural ecosystems corresponds to species-level differences or simply intraspecific (strainstrain) variability. Our conceptualization of guilds of ecologically similar protistan taxa is not meant to challenge the ecological species concept (or any other species concept) for protists by suggesting that different species of protists are exact ecological duplicates of other species. However, many protists appear to have overlapping abilities (e.g. in the use of inorganic nutrients for phototrophs or prey types for heterotrophs). Arguments of the species concept aside, any degree of genetic dissimilarity that imparts different competitive advantages could allow for rapid commu- 


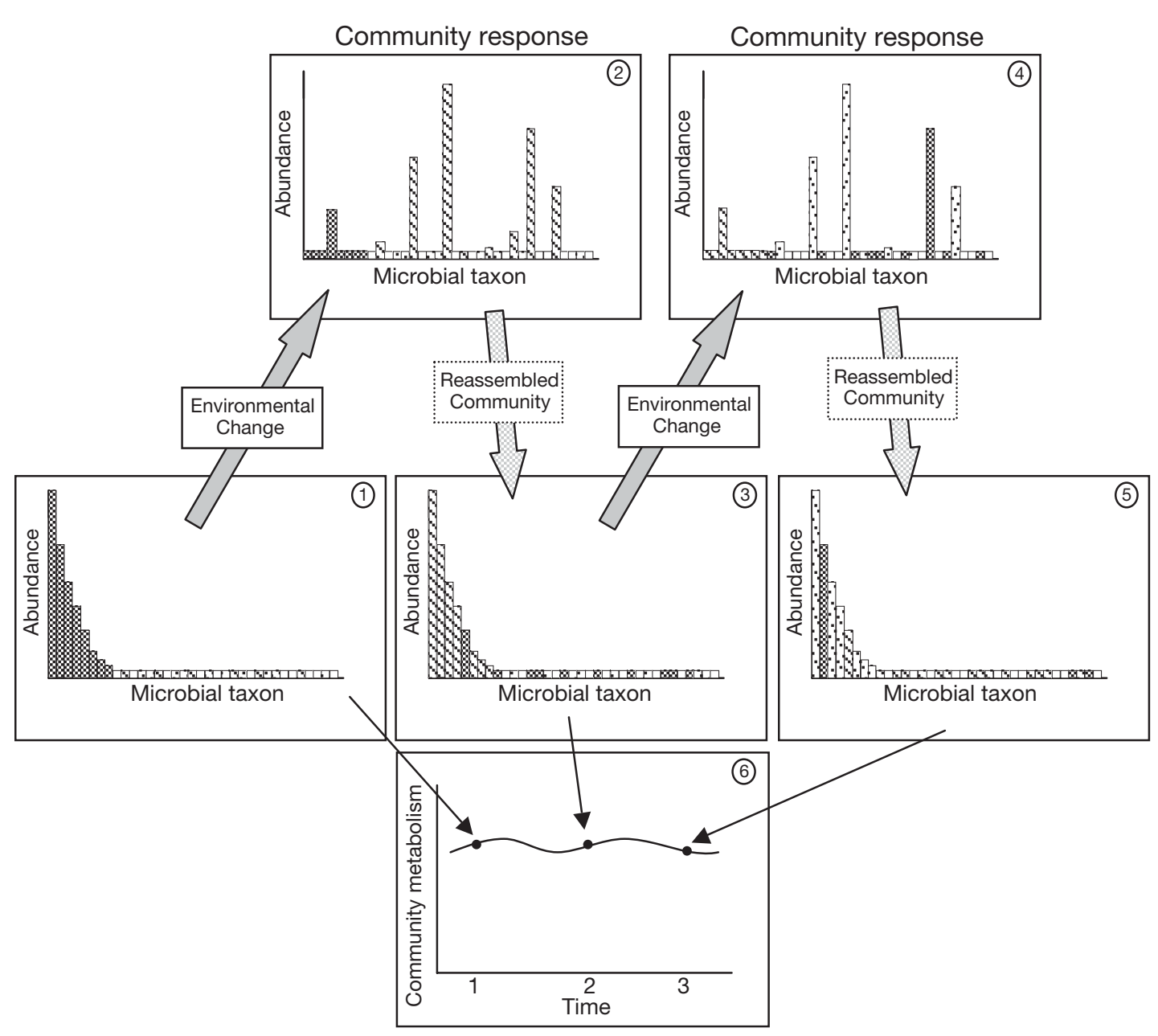

Fig. 2. Hypothesis of protistan community structure and function, rapid community reassembly and the importance of the rare biosphere of microbes. A characteristic rank abundance curve of a hypothetical microbial community (1) affected by changing environmental conditions results in rapid changes in the population abundances of specific taxa, with many of the favored species among the rare biosphere (2). The reassembled community exhibits the same shape to its rank abundance curve when sorted by abundance (3), albeit with different common and rare taxa. A subsequent change in environmental conditions results in another succession of taxa (4), again giving rise to a rank abundance curve of similar shape but different taxonomic composition (5). While substantial changes in community structure have taken place $(1,3,5)$, it is hypothesized that changes in community function as a consequence of environmental perturbation may be minimal (6). The approximate time frame for significant community reassembly may be as short as $1 \mathrm{~d}$ (Countway et al. 2005)

nity reassembly without major changes in community processes.

Although the scenario described above implies that individual taxa may be substituted independently, it is equally probable (perhaps more probable) that groups of species linked by ecological activity may become dominant, or rare, as a single unit. Species that are functionally linked (e.g. those involved in metabolic consortia or symbiotic relationships) and have the same chemical and physical tolerances may undergo simultaneous success or failure. If true, this scenario presents a testable hypothesis in that network analysis of microbial species should reveal such relationships. Indeed, such analyses are beginning to reveal the exis- tence of discernible, temporally repeating networks among microbial taxa (Fuhrman et al. 2006, Fuhrman et al. 2009).

The role of the rare biosphere hypothesized here does not exclude other possible reasons for the existence and persistence of microbial taxa at very low abundance in an ecosystem. For example, many apex predators persist at low abundance and play important ecological roles in biological communities at those low abundances. However, the role of the rare biosphere proposed here for microbial taxa is consistent with observations of the ubiquity of this component of microbial communities, and rapid changes in community composition as noted in the following section. 


\section{PROTISTAN RARE BIOSPHERE IN ACTION: SOME EXAMPLES}

A growing database of observations and experimental outcomes is consistent with the scenario detailed in Fig. 2 regarding the composition and role of the rare biosphere. This information has not been gathered with the specific intent of testing the hypothesis for an ecological role of rare taxa. Nevertheless, these data offer insights into the composition, persistence and potential ecological importance of rare protistan species.

\section{Culturing the protistan rare biosphere}

Traditional approaches used to establish laboratory cultures of microbes have inadvertently provided proof of the presence of many rare taxa in nature. Most culture media and conditions are highly selective and create environmental settings that are substantially different than those in most natural ecosystems. Interestingly, the species that have been successfully cultured have often been taxa that are present at very low abundance in nature. This situation is well known for bacterial assemblages in aquatic ecosystems, where many of the cultured strains have been shown to be present at very low abundance when assayed using culture-independent approaches (Pedrós-Alió 2006, Pedrós-Alió 2007). This realization has stimulated active research to improve our knowledge of both common and rare microbial species that have not yet been brought into laboratory culture (Zengler 2008).

Culture approaches have also been effective at enriching rare taxa from natural protistan assemblages, although our ability to enrich and culture rare protistan taxa is probably not as explicitly recognized or acknowledged as it has been for bacteria (Caron \& Gast 2008). A classic example is the observation that species of the heterotrophic flagellate genus Paraphysomonas appear to be globally distributed because they can be easily enriched from natural water samples collected from a wide variety of locales (Finlay \& Clarke 1999a). These species often dominate enrichment cultures established by stimulating the bacterial assemblages of samples with organic compounds (Lim et al. 1999). Paraphysomonas species typically grow readily on the bacteria growing in these enrichments, and while they often become the dominate protists in these enrichment cultures after several days, their abundances typically are so low in unenriched water samples that they cannot be detected using species-specific probing methods (Fig. 3). Species-specific probing methods are useful because they can be used to track microbial taxa as they wax and wane in abundance and thereby provide insight into the activities of rare protistan taxa.

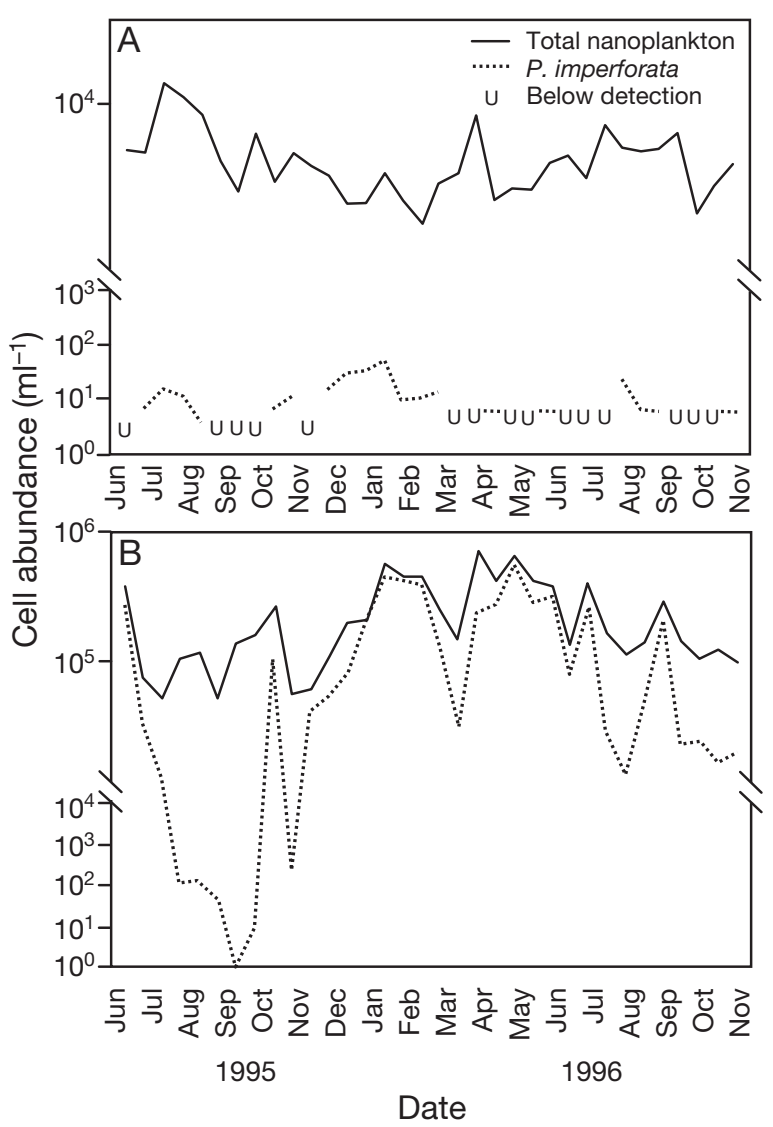

Fig. 3. Paraphysomonas imperforata. Demonstration of dramatic changes in the abundance of a heterotrophic, bacterivorous flagellate ( $P$. imperforata) from rare (or undetectable, U) to dominant in highly selective culture conditions. Organic substrates were added to water samples to enrich the natural bacterial assemblages. Samples preserved (A) immediately upon collection and (B) following several days of incubation were assayed for the abundances of total nanoplankton (-) and $P$. imperforata $(\cdots)$ by fluorescence in situ hybridization. Enrichment of the bacteria resulted in substantial increases in the abundance of $P$. imperforata for all samples (even when the species was undetectable in the unenriched sample) and dominance of the total nanoplanktonic protists by this flagellate in approximately half of the samples. Note the log scale (and break) in the $y$-axis. Data redrawn from Lim et al. (1999)

\section{Compositional changes during incubation}

Studies involving the incubation of water samples under conditions that mimic natural conditions have demonstrated that changes in the dominant taxa of microbial eukaryotes take place in a manner similar to the well-known bottle effect for bacteria (Zobell 1943). Water collected from the North Atlantic and incubated at ambient temperature and light experienced dramatic changes in community structure after $1 \mathrm{~d}$ of incubation, and further reassembly after 3 d (Fig. 4; adapted from Countway et al. 2005). A total of 970 

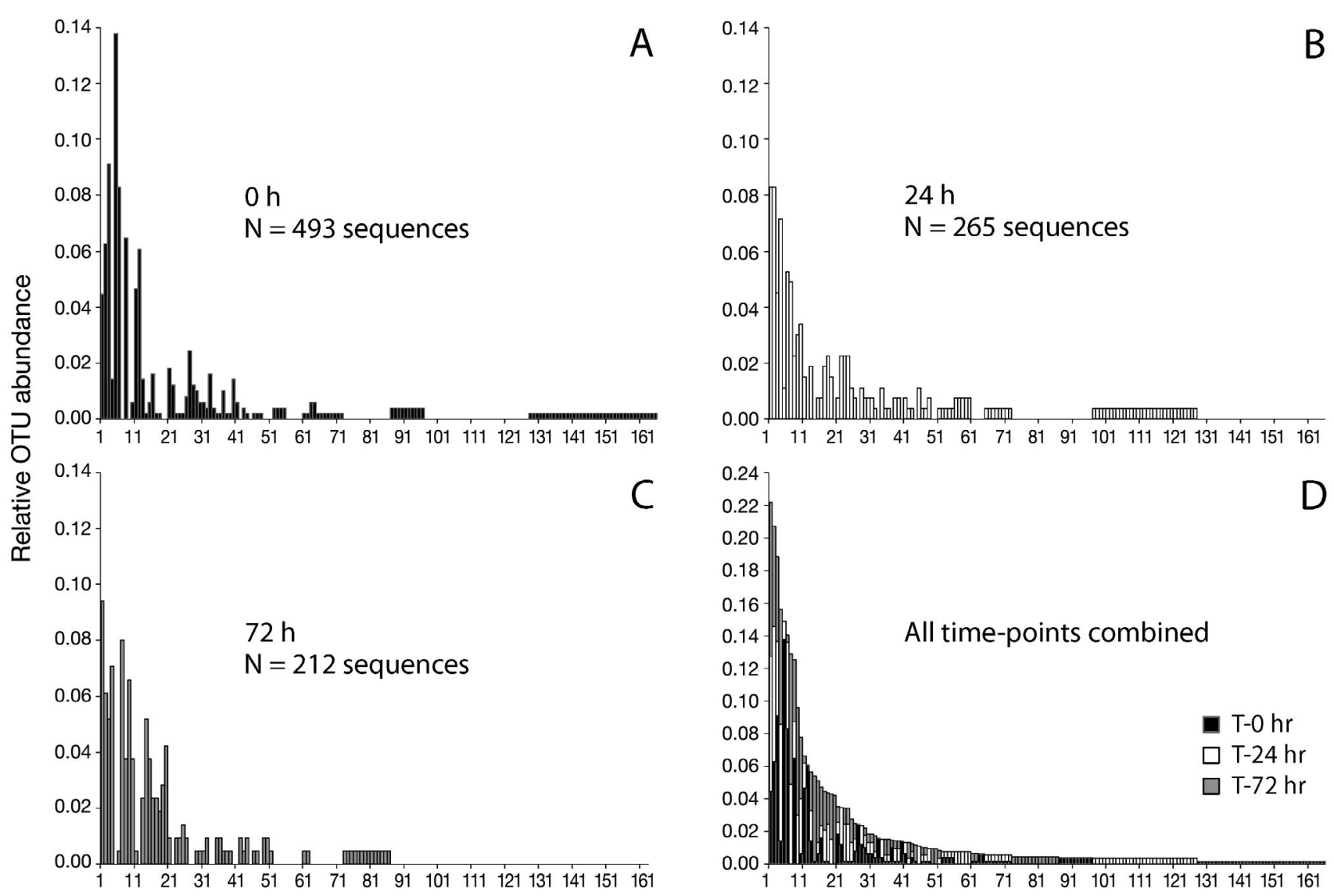

\section{OTU rank}

Fig. 4. Evidence for rapid community reassembly among microbial eukaryotes as a consequence of minor environmental perturbation. Results from a bottle incubation experiment performed using water collected from the North Atlantic. Clone libraries $(18 \mathrm{~S})$ were established and sequenced from a water sample (A) at the beginning of the incubation period and at intervals of (B) 24 and (C) $72 \mathrm{~h}$ under conditions of ambient temperature and light. Data from all 3 time-points are depicted in (D). Rank abundances for each operational taxonomic unit (OTU) are shown along the same scale of OTUs for each time-point in all panels. Note that most of the rare taxa (right side of each panel) were observed only in 1 of the 3 time points. Details of sampling, experimental setup and DNA collection, sequencing and processing are given in Countway et al. (2005). Data in (D) redrawn from Countway et al. (2005)

rDNA (18S) sequences yielded 165 different operational taxonomic units (OTUs) established at approximately species-level distinctions based on an automated program described in Caron et al. (2009). Briefly, the Microbial Eukaryote Species Assignment (MESA) program performs automated comparisons of $18 \mathrm{~S}$ sequences to assign sequences from environmental sequence data sets to OTUs. The program was developed using $18 \mathrm{~S}$ sequences from morphologically defined protistan taxa to establish an approximately species-level sequence similarity value that is applied to environmental data sets.

Only $18 \%$ of all OTUs observed in the water sample by Countway et al. (2005) were detected at all 3 sampling intervals, while $65 \%$ were detected at only 1 of the 3 sampling times. Most of the unique OTUs were present as rare taxa (Fig. 4); however, a number of taxa that were rare (or below detection) at the initial sampling time appeared as abundant taxa at subsequent sampling intervals and vice versa. These results demonstrate that even subtle shifts in environmental conditions can bring about rapid changes in the relative abundances of microbial eukaryotic taxa.

Can community reassembly in nature happen rapidly enough among protistan taxa such that community activity might be preserved? Recent evidence suggests that the answer is 'yes'. Observations of natural assemblages over short temporal or spatial scales indicate that rapid changes in composition may be the norm for protistan assemblages. Vigil et al. (2009) employed terminal restriction fragment length polymorphism (T-RFLP) analysis to demonstrate that natural assemblages of microbial eukaryotes exhibited different dominant taxa in neighboring bays on the 
east coast of the US sampled at approximately the same time and over short time intervals (several days) within each bay. Relatively rapid temporal shifts in the dominant phototrophic protists in phytoplankton assemblages have been documented (Karentz \& Smayda 1998), but the speed at which these changes take place has not been widely recognized. Even less is known regarding the rates of community compositional changes involving heterotrophic protists and most small, morphologically nondescript protistan taxa.

\section{Similarity among communities from geographically distant locations}

The results of Vigil et al. (2009) indicate the potential for the existence of highly divergent protistan community compositions from habitats that are separated by relatively small distances or short time periods. On the other hand, one might expect greater similarity between assemblages existing under similar environmental conditions but separated by great distances than between neighboring assemblages exposed to different environmental conditions if the protistan rare biosphere is large and ubiquitous (or nearly so), as hypothesized. Indeed, evidence is now emerging that supports this prediction (Fig. 5).

Microbial eukaryote assemblages surveyed from samples collected in the North Atlantic and North Pacific revealed a greater degree of similarity among samples collected from deep water ( $\geq 150 \mathrm{~m}$ ) or the euphotic zone (solid lines, Fig. 5) relative to samples collected at shallow and deep locations within a single ocean basin. Additionally, seasonal differences among the protistan assemblages of the euphotic zone from the North Pacific were substantial, and resulted in greater similarity between some Pacific and Atlantic samples within the euphotic zone, compared to similarities between all of the samples collected from the shallow Pacific (dotted lines inside the right-hand ellipse, Fig. 5). These findings appear to support the hypothesis that a large rare biosphere containing many shared taxa exists globally, and that selective processes acting locally are a dominant factor in establishing the taxonomic composition of the dominant microbial assemblage. In short, extremely con- stant and/or highly selective environments might be expected to have more similar community compositions than adjacent habitats with differing environmental conditions.

Results also exist that are consistent with the supposition that groups, or guilds, of protists are selected together during community reassembly. The existence of co-occurrence among species (species co-occurring more or less frequently than expected by chance) previously applied to communities of macroorganisms has recently been reported for microorganismal communities as well (Horner-Devine et al. 2007). These relationships may be the result of predation, mutualism, competition, syntrophy and other interactions among micro-organisms. 
What do these results mean in terms of rates of community processes (growth, grazing, mortality and elemental cycling) during community reassembly? Answering that question will require experimental studies that couple characterizations of community structure (including the rare biosphere) with measurements of community function. Such studies will test the hypothesis that the rare biosphere plays a fundamental role in buffering community function, and yield useful information to improve predictive models of microbial community response to changing environmental conditions. Ecologists have begun to experimentally examine the relationship between biological diversity and the stability, resilience or predictability of community function (McGrady-Steed et al. 1997, Naeem \& Shibin 1997). This work shows great promise for generating a theoretical underpinning of the relationship between diversity, ecological redundancy and ecosystem function. Hopefully, future iterations of these simplistic studies will take into account the huge diversity of rare microbial taxa.

Additionally, if the hypothesis of ecologically redundant, functional guilds of species within the microbial rare biosphere is true, then bottle experiments that characterize the rare biosphere through time may help differentiate those species that are present as rare taxa because they are persistent in an ecosystem (i.e. do not require continual reintroduction via dispersal from other environments) from those that are dependent on reintroduction. Such information may provide insight into how the existence of some of the rare biosphere taxa is maintained.

\section{COMMUNITY RESTRUCTURING IN A CHANGING WORLD}

\section{Functional resilience of microbial communities}

The concept of a rare biosphere of microbes that is present in nearly all natural ecosystems is just now emerging as a new paradigm within the microbial ecological scientific community, although the enormous diversity of microbes has been evident for many decades. The supposition that microbial communities are constantly reassembling may at first seem trivial because it is well known that species dominance is quite different in many microbial communities. However, we suggest that the importance of the rare biosphere as a mechanism for backfilling ecosystem function in the face of changing environmental conditions is not trivial. Indeed, the existence of an enormous rare biosphere may represent a feature that is unique to microbial communities (relative to macrobial communities) and a possible explanation for the biogeochem- ical dominance of microbes for billions of years on our planet (Gould 1996).

The concept and hypothesis described here imply that many microbial taxa occur in all environments, albeit at vanishingly low abundances, yet they are capable of stepping in to play important ecological roles if environmental conditions change to create a selective advantage. Does this mean that we do not need to worry about losing microbial diversity as a consequence of changing climate? To a large degree, the answer to that question might be 'yes'. Climatic conditions during Earth's history have spanned a tremendous range and have selected for the evolution of a very wide diversity of microbes. If the complete global extinction of microbial species is exceedingly difficult, as suggested here, then the persistence of many of these species as components of the rare biosphere could provide biological buffering capacity, i.e. a reservoir of microbial species that might be responsive to most of the possible climatic scenarios on our planet. Taxonomic composition might differ greatly from microbial assemblages that currently dominate, yet they may be capable of maintaining basic biogeochemical cycles in the face of massive extinctions of macroscopic taxa.

\section{How much change is acceptable?}

If the rare biosphere plays the ecological role proposed herein, then gross community-level processes (e.g. primary production, trophic interactions, community respiration) probably would be relatively conserved even with substantial changes in environmental forcing and dramatic reassemblies of the dominant microbial taxa. However, the particular species conducting these processes in the reassembled communities might have important implications for human activities and well-being. For example, relatively minor changes in environmental factors might lead to extensive changes in the dominance of toxic, noxious or otherwise undesirable species of algae (e.g. harmful algal bloom-forming species of algae, or weed species that strongly dominate and greatly dampen species evenness within the community). In turn, selection for even one or a few of these species may escalate into major transformations in food web structure, creating widespread changes in selective advantages and disadvantages for many other species, even if rates of elemental cycling and energy flow remain relatively constant.

Changes in microbial community structure do not have to be cataclysmic to be deleterious. Recurrent blooms of 2 noxious pelagophyte algae along the coasts of the Middle Atlantic US and lagoons of Texas 
during the past few decades (so-called brown tides) had widespread effects on benthic macrophytes and filter-feeding bivalves as well as planktonic micro- and macrozooplankton (Bricelj \& Lonsdale 1997, Buskey et al. 2001, Gobler et al. 2005). In a similar fashion, overfishing of several commercially valued macroscopic species has resulted in ecological extinction of these species (although not necessarily biological extinction), significantly altering food web structure and pathways of energy flow in many marine coastal ecosystems (Pandolfi et al. 2005, Worm et al. 2006). Removal of key predatory species has allowed prey species to flourish, resulting in what many have referred to as 'the rise of slime': the success of undesirable species that greatly alter pathways of energy flow through marine ecosystems. There is little argument that these changes in the structure of the biological community are harmful, yet there is little evidence in these examples to suggest that basic biogeochemical processes have been severely altered or curtailed.

This supposition is not to say that microbial species diversity cannot be reduced and microbiological activities cannot be curtailed. The mechanisms and potential consequences of microbial species extinctions have been considered by Weinbauer \& Rassoulzadegan (2007). Mass extinctions of macrobial species via cataclysmic events on Earth such as meteor strikes, or the removal of individual species via natural selection or human activities, could be expected to reduce overall microbial species diversity by eliminating unique microhabits for mutualisitic or parasitic microbes. The significance of these microbial extinctions are unknown.

Finally, if environmental changes exceed the capacity for physiological and/or behavioral adaptation collectively contained within the microbial taxa comprising the rare biosphere, then biogeochemical cycles and processes might change dramatically or cease altogether. Scenarios of this sort may have taken place on other planets that once supported life. The presence of compounds on Mars that may represent biosignatures of past life have fueled an active debate regarding the possibility of past life on that planet (ThomasKeprta et al. 2001, Formisano et al. 2004).

At what point would we expect changes in environmental conditions to bring about wholesale changes in microbial community function? That is a difficult question to answer, and the hypothesis proposed herein is presently difficult to confirm or refute, for at least 2 reasons. (1) Our understanding of the depths of microbial diversity is still rudimentary for most natural ecosystems. Although high-throughput sequencing approaches are rapidly changing this situation, it will still be some time before we fully understand the taxonomic identity and functional significance of the sequence diversity that we are now uncovering within the rare biosphere of natural microbial assemblages. (2) Divergent views presently exist regarding the dispersal of microbial species on our planet, and this knowledge is fundamental for understanding the role of dispersal in maintaining a global microbial rare biosphere. Endemism and extinction are accepted as paradigms in the ecology and evolution of many macrobial species, but these issues are much less clear for most microbial species. Opinions among biologists differ regarding the size at which organisms are no longer easily transported around the world, and therefore exhibit endemism (Finlay \& Clarke 1999b, Finlay 2002, Fenchel \& Finlay 2004, Fenchel 2005). Interestingly, protistan species exist across a size range that spans the size at which organisms might be expected to exhibit limited dispersal (a rough approximation of $1 \mathrm{~mm}$ has been suggested; Fenchel \& Finlay 2004). If effective dispersal is a major factor explaining the existence of the rare biosphere of microbes in nature, then one might expect community processes carried out by assemblages of large organisms (down to the size of some protistan taxa) to be less resilient than those performed by assemblages of smaller organisms. Generalizing broadly, one might postulate that the number of rare taxa in an assemblage might be inversely related to organismal size (Fenchel \& Finlay 2004), and functional resilience of the community might be directly related to the number of rare taxa. Resolution of these suppositions is presently thwarted by disagreements or lack of information regarding dispersal rates of microbes, as well as debates over the species concept (Caron 2009).

\section{CONCLUSIONS}

The protistan rare biosphere is a dynamic collection of an apparently vast number of taxa with potentially widespread distributions and presently unknown ecological significance. Circumstantial evidence indicates that the rare biosphere may comprise a source of ecological redundancy that maintains continued ecosystem function in the face of rapid and frequent shifts in the taxonomic composition of the dominant taxa brought about by changing environmental conditions. Microbial ecologists have only begun to characterize the extent of microbial diversity in nature, and few direct experimental measurements have been performed to examine the relationship between the rare biosphere and resilience and/or stability of community function. These studies must begin in earnest as we strive to understand the relationship between the immense diversity contained within the rare biosphere and the manner in which these taxa affect the ecosystem processes of biological communities. 
Acknowledgements. The preparation of this manuscript was supported in part by grants from the Gordon and Betty Moore Foundation and the National Science Foundation (MCB0703159 and OCE-0550829).

\section{LITERATURE CITED}

Agis M, Granda A, Dolan JR (2007) A cautionary note: examples of possible microbial community dynamics in dilution grazing experiments. J Exp Mar Biol Ecol 341:176-183

Alpermann TJ, Beszteri B, John U, Tillmann U, Cembella AD (2009) Implications of life-history transitions on the population genetic structure of the toxigenic marine dinoflagellate Alexandrium tamarense. Mol Ecol 18:2122-2133

Boenigk J, Pfandl K, Stadler P, Chatzinotas A (2005) High diversity of the 'Spumella-like' flagellates: an investigation based on the SSU rRNA gene sequences of isolates from habitats located in six different geographic regions. Environ Microbiol 7:685-697

Bricelj WM, Lonsdale DJ (1997) Aureococcus anophagefferens: causes and ecological consequences of brown tides in U.S. Mid-Atlantic coastal waters. Limnol Oceanogr 42: 1023-1038

Buskey EJ, Liu H, Collumb C, Guilherme J, Bersano JGF (2001) The decline and recovery of a persistent Texas brown tide algal bloom in the Laguna Madre (Texas, USA). Estuaries 24:337-346

Caron DA (2000) Protistan herbivory and bacterivory. In: Paul $\mathrm{J}$ (ed) Marine microbiology: methods in microbiology, Vol 30. Academic Press, London, p 289-315

> Caron DA (2009) Protistan biogeography: Why all the fuss? J Eukaryot Microbiol 56:105-112

Caron DA, Gast RJ (2008) The diversity of free-living protists: seen and unseen, cultured and uncultured. In: Zengler K (ed) Accessing uncultivated microorganisms: from the environment to organisms and genomes and back. ASM Press, Washington, DC, p 67-93

Caron DA, Goldman JC, Fenchel T (1990) Protozoan respiration and metabolism. In: Capriulo GM (ed) Ecology of marine protozoa. Oxford University Press, New York, p 307-322

Caron DA, Countway PD, Savai P, Gast RJ and others (2009) Defining DNA-based operational taxonomic units for microbial eukaryote ecology. Appl Environ Microbiol 75: 5797-5808

Countway PD (2005) Molecular ecology of marine protistan assemblages. University of Southern California, Los Angeles, CA

Countway PD, Gast RJ, Savai P, Caron DA (2005) Protistan diversity estimates based on 18S rDNA from seawater incubations in the western North Atlantic. J Eukaryot Microbiol 52:95-106

> Countway PD, Gast RJ, Dennett MR, Savai P, Rose JM, Caron DA (2007) Distinct protistan assemblages characterize the euphotic zone and deep sea $(2500 \mathrm{~m})$ of the western North Atlantic (Sargasso Sea and Gulf Stream). Environ Microbiol 9:1219-1232

DeLong EF (1992) Archaea in coastal marine environments. Proc Natl Acad Sci USA 89:5685-5689

> Dolan JR (1991) Guilds of ciliate microzooplankton in the Chesapeake Bay. Estuar Coast Shelf Sci 33:137-152

Dolan JR, McKeon K (2005) The reliability of grazing rate estimates from dilution experiments: Have we over-estimated rates of organic carbon consumption by microzooplankton? Ocean Sci 1:1-7

Dolan JR, Ritchie ME, Tunin-Ley A, Pizay MD (2009) Dynam- ics of core and occasional species in the marine plankton: tintinnid ciliates in the north-west Mediterranean Sea. J Biogeogr 36:887-895

> Elshahed MS, Youssef NH, Spain AM, Sheik C and others (2008) Novelty and uniqueness patterns of rare members of the soil biosphere. Appl Environ Microbiol 74: 5422-5428

Epstein S, López-García P (2008) 'Missing' protists: a molecular perspective. Biodivers Conserv 17:261-276

Evans KM, Kühn SF, Hayes PK (2005) High levels of genetic diversity and low levels of genetic differentiation in North Sea Pseudo-nitzschia pungens (Bacillariophyceae) populations. J Phycol 41:506-514

Falkowski PG, Raven JA (1997) Aquatic photosynthesis. Blackwell Scientific, Oxford

- Fenchel T (2005) Cosmopolitan microbes and their 'cryptic' species. Aquat Microb Ecol 41:49-54

- Fenchel T, Finlay BJ (1983) Respiration rates in heterotrophic, free-living protozoa. Microb Ecol 9:99-122

> Fenchel T, Finlay BJ (2003) Is microbial diversity fundamentally different from biodiversity of larger animals and plants? Eur J Protistol 39:486-490

> Fenchel T, Finlay BJ (2004) The ubiquity of small species: patterns of local and global diversity. Bioscience 54:777-784

Ferrier-Pagès C, Rassoulzadegan F (1994) N remineralization in planktonic protozoa. Limnol Oceanogr 39:411-419

> Finlay BJ (2002) Global dispersal of free-living microbial eukaryote species. Science 296:1061-1063

Finlay BJ, Clarke KJ (1999a) Apparent global ubiquity of species in the protist genus Paraphysomonas. Protist 150:419-430

- Finlay BJ, Clarke KJ (1999b) Ubiquitous dispersal of microbial species. Nature 400:828

Finlay BJ, Esteban G, Fenchel T (2004) Protist diversity is different? Protist 155:15-22

Foissner W (2006) Biogeography and dispersal of microorganisms: a review emphasizing protists. Acta Protozool 45:111-136

- Formisano V, Atreya S, Encrenaz T, Ignatiev N, Giuranna M (2004) Detection of methane in the atmosphere of Mars. Science 306:1758-1761

Fuhrman JA, McCallum K, Davis AA (1992) Novel major archaebacterial group from marine plankton. Nature 356:148-149

Fuhrman JA, Hewson I, Schwalbach MS, Steele JA, Brown MV, Naeem S (2006) Annually reoccurring bacterial communities are predictable from ocean conditions. Proc Natl Acad Sci USA 103:13104-13109

Fuhrman JA, Steele J, Sun F, Xia L, Countway PD, Caron DA (2009) Association network analysis shows potential marine food web interactions. American Society of Limnology and Oceangraphy, Aquatic Sciences Meeting, 25-30 Jan 2009, Nice

Gobler CJ, Lonsdale DJ, Boyer GL (2005) A review of the causes, effects, and potential management of harmful brown tide blooms caused by Aureococcus anophagefferens (Hargraves et Sieburth). Estuaries 28:726-749

Gould SJ (1996) Planet of the bacteria. Washington Post Horizon, Washington, DC, p 344

Green J, Bohannan BJM (2006) Spatial scaling of microbial biodiversity. Trends Ecol Evol 21:501-507

> Green JL, Holmes AJ, Westoby M, Oliver I and others (2004) Spatial scaling of microbial eukaryote diversity. Nature 432:747-750

Horner-Devine MC, Silver JM, Leibold MA, Bohannan BJM and others (2007) A comparison of taxon co-occurrence patterns for macro- and microorganisms. Ecology 88: 
$1345-1353$

Huse SM, Dethlefsen L, Huber JA, Welch M, Relman DA, Sogin ML (2008) Exploring microbial diversity and taxonomy using SSU rRNA hypervariable tag sequencing. PLoS Genet 4:e1000255

Karentz DK, Smayda TJ (1998) Temporal patterns and variations in phytoplankton community organization and abundance in Narragansett Bay during 1959-1980. J Plankton Res 20:145-168

Landry MR, Kirshtein J, Constantinou J (1995) A refined dilution technique for measuring the community grazing impact of microzooplankton, with experimental tests in the central equatorial Pacific. Mar Ecol Prog Ser 120: $53-63$

Lim EL, Caron DA, Dennett MR (1999) The ecology of Paraphysomonas imperforata based on studies employing oligonucleotide probe identification in coastal water samples and enrichment culture. Limnol Oceanogr 44:37-51

Lovejoy C, Massana R, Pedrós-Alió C (2006) Diversity and distribution of marine microbial eukaryotes in the Arctic Ocean and adjacent seas. Appl Environ Microbiol 72: 3085-3095

Massana R, Guillou L, Diez B, Pedrós-Alió C (2002) Unveiling the organisms behind novel eukaryotic ribosomal DNA sequences from the ocean. Appl Environ Microbiol 68:4554-4558

McGrady-Steed J, Harris PM, Morin PJ (1997) Biodiversity regulates ecosystem predictability. Nature 390:162-165

> Naeem S, Shibin L (1997) Biodiversity enhances ecosystem stability. Nature 390:507-509

Pandolfi JM, Jackson JBC, Baron N, Bradbury RH and others (2005) Are US coral reefs on the slippery slope to slime? Science 307:1725-1726

Pedrós-Alió C (2006) Microbial diversity: Can it be determined? Trends Microbiol 14:257-263

Pedrós-Alió C (2007) Dipping into the rare biosphere. Science 315:192-193

Romari K, Vaulot D (2004) Composition and temporal variability of picoeukaryote communities at a coastal site of the English Channel from 18S rDNA sequences. Limnol Oceanogr 49:784-798

Rynearson TA, Armbrust EV (2004) Genetic differentiation among populations of the planktonic marine diatom Ditylum brightwellii (Bacillariophyceae). J Phycol 40:34-43

Submitted: May 18, 2009; Accepted: July 10, 2009
Rynearson TA, Newton JA, Armbrust EV (2006) Spring bloom development, genetic variation and population succession in the planktonic diatom Ditylum brightwellii. Limnol Oceanogr 51:1249-1261

Schloss PD, Handelsman J (2004) Status of the microbial census. Microbiol Mol Biol Rev 68:686-691

Shankle AM, Mayali X, Franks PJS (2004) Temporal patterns in population genetic diversity of Prorocentrum micans (Dinophyceae). J Phycol 40:239-247

Sherr EB, Sherr BF (2002) Significance of predation by protists in aquatic microbial food webs. Antonie Leeuwenhoek 81:293-308

Sogin ML, Morrison HG, Huber JA, Welch DM and others (2006) Microbial diversity in the deep sea and the underexplored 'rare biosphere'. Proc Natl Acad Sci USA 103:12115-12120

Stoeck T, Taylor GT, Epstein SS (2003) Novel eukaryotes from the permanently anoxic Cariaco Basin (Caribbean Sea). Appl Environ Microbiol 69:5656-5663

> Stoeck T, Zuendorf A, Behnke A, Breiner HW (2007) A molecular approach to identify active microbes in environmental eukaryote clone libraries. Microb Ecol 53:328-339

Thomas-Keprta KL, Clemett SJ, Bazylinski DA, Kirschvink JL and others (2001) Truncated hexa-octahedral magnetite crystals in ALH84001: presumptive biosignatures. Proc Natl Acad Sci USA 98:2164-2169

> Vaulot D, Eikrem W, Viprey M, Moreau H (2008) The diversity of small eukaryotic phytoplankton $(<3 \mu \mathrm{m})$ in marine ecosystems. FEMS Microbiol Rev 32:795-820

- Vigil P, Countway PD, Rose JM, Gobler CJ, Lonsdale DJ, Caron DA (2009) Rapid shifts in dominant taxa among microbial eukaryotes in estuarine ecosystems. Aquat Microb Ecol 54:83-100

> Weinbauer MG, Rassoulzadegan F (2007) Extinction of microbes: evidence and potential consequences. Endang Species Res 3:205-215

Worm B, Barbier EB, Baumont N, Duffy JE and others (2006) Impacts of biodiversity loss on ocean ecosystem services. Science 314:787-790

Zengler K (2008) Accessing uncultivated microorganisms: from the environment to organisms and genomes and back. ASM Press, Washington, DC

Zobell CE (1943) The effect of solid surfaces upon bacterial activity. J Bacteriol 46:39-56

Proofs received from author(s): September 29, 2009 